\title{
Modeling Human Body Using Four-Pole Debye Model in Piecewise Linear Recursive Convolution FDTD Method for the SAR Calculation in the Case of Vehicular Antenna
}

\author{
Ammar Guellab $(D)$ and Qun Wu \\ Harbin Institute of Technology, Xidazhi Street, Harbin, China \\ Correspondence should be addressed to Ammar Guellab; ammarguellab400@hit.edu.cn and Qun Wu; qwu@hit.edu.cn
}

Received 16 September 2017; Revised 7 December 2017; Accepted 28 December 2017; Published 19 April 2018

Academic Editor: Rodolfo Araneo

Copyright (C) 2018 Ammar Guellab and Qun Wu. This is an open access article distributed under the Creative Commons Attribution License, which permits unrestricted use, distribution, and reproduction in any medium, provided the original work is properly cited.

\begin{abstract}
We propose an efficient finite difference time domain (FDTD) method based on the piecewise linear recursive convolution (PLRC) technique to evaluate the human body exposure to electromagnetic (EM) radiation. The source of radiation considered in this study is a high-power antenna, mounted on a military vehicle, covering a broad band of frequency $(100 \mathrm{MHz}-3 \mathrm{GHz})$. The simulation is carried out using a nonhomogeneous human body model which takes into consideration most of the internal body tissues. The human tissues are modeled by a four-pole Debye model which is derived from experimental data by using particle swarm optimization (PSO). The human exposure to EM radiation is evaluated by computing the local and whole-body average specific absorption rate (SAR) for each occupant. The higher in-tissue electric field intensity points are localized, and the SAR values are compared with the crew safety standard recommendations. The accuracy of the proposed PLRC-FDTD approach and the matching of the Debye model with the experimental data are verified in this study.
\end{abstract}

\section{Introduction}

Nowadays, the battlefield is making more use of the electromagnetic spectrum to satisfy diverse operational needs that range from high-rate tactical links to broadband jammers. This calls for the use of vehicular antennas that transmit high powers, putting the life of crew personnel at stake through high electromagnetic (EM) exposure [1]. Such high-power radiation can occur in a broad frequency band (HF, VHF, and UHF). IEEE Technical Committee 95 (IEEE-TC95) proposed a standard for military workplaces whose purpose is to provide exposure limits to assure the personnel safety in a military workplace and provide protection against unfavorable effects of the electromagnetic radiation on the human body [2]. This standard expresses its recommendations as dosimetry reference limits (DRLs) which can be expressed by the within-tissue electric field strength or the specific absorption rate (SAR).

With the aim of protecting the crew against high-power EM radiation, much research is devoted to compute the level of the induced SAR from various EM sources. Accurate and efficient computational methods are sought after for this purpose [3-6]. This can be so challenging and complicated due to the human body and the vehicle structure complexity especially when a broad frequency band is considered.

A numerical simulation for the computation of the SAR in vehicle passengers due to an onboard $900 \mathrm{MHz}$ transmission system is carried out in [3]. The FDTD method is used for the analysis of the SAR from a cell phone inside a vehicle in [4]. However, those studies are concerned with a single narrow band of frequency. Diao [5] uses the FDTD method for the estimation of the induced SAR from multiple communication devices, which occupy different frequencies inside the vehicle. In [6] the FDTD simulation of a complex structure vehicle is carried out to investigate the effect of the passenger's number on the SAR level for many frequencies.

The previously cited works obtain a rigorous estimation for the SAR level induced by multiple sources. Nevertheless, they focus on isolated and narrow bands of frequency and use human body models that do not take into account the 
frequency dependency of electric characteristics of the human tissues.

A frequency-dependent model for the human tissue has been provided by Gabriel et al. [7], which is based on a four-pole Cole-Cole equation. This model leads to a highly precise evaluation of the complex electric characteristics (permittivity and conductivity) of the dispersive media constituting the human tissue. Nevertheless, the complexity of this model makes it disadvantageous for the implementation of time-domain numerical simulation.

Gabriel's Cole-Cole model is reduced to a two-pole Debye model by using the least squares fitting technique in $[8,9]$. The two-pole Debye tissue model is more suitable for the time domain methods but is less accurate than the Cole-Cole model. In [10], a fourth-order Debye model is used in the convolutional-based FDTD for the modeling of the electromagnetic waves propagation in the human head tissues. Based on this study, the fourth-order Debye model is more accurate than the two-pole Debye model and is simpler than the Cole-Cole model. Therefore, it combines the high accuracy of computations and the simplicity of implementation.

Despite the fact that the high-order Debye model matches perfectly with the experimental dielectric characteristics, it leads to computational complexity and an accumulative numerical dispersion. In the literature, many works are devoted to the development of highly accurate methods for frequency-dependent media. In [10], a general recursive convolution FDTD is applied for the simulation of the fourth-order Debye model. However, this method is less accurate. In [11] the alternating direction implicit finite difference time domain (ADI-FDTD) method is extended to the simulation of the Debye dispersive media. The advantage of the ADI-FDTD is its unconditional stability. However, it suffers from the loss of precision. In [12], a simple trapezoidal recursive (TRC) technique is used in the FDTD analysis of frequency dependent media, where it is proven that the TRC method is more accurate than the RC technique. For more accuracy, a piecewise linear recursive convolution (PLRC) technique is used in [13]. The PLRC technique is an efficacious method for dealing with the dispersive models (Debye, Lorentz, and Drude) [13].

In this work, a highly accurate four-pole Debye model is developed, which perfectly fits with the experimental data [14] of the whole-body tissues in a broad band of frequency ranging from $100 \mathrm{MHz}$ to $3 \mathrm{GHz}$. For this purpose, we applied the particle swarm optimization (PSO) algorithm for the optimization role. Then, we proposed an implementation of a three-dimension FDTD based on the piecewise linear recursive convolution (PLRC) technique for the simulation of the four-pole Debye model. The agreement of the Debye model with the experimental dielectric properties of each tissue of the human body and the accuracy of the proposed FDTD method are verified.

After that, a typical military vehicle, commonly known as (Humvee) is considered. It is equipped with a large antenna used for radio-transmission applications. A reduced complexity model is used for the vehicle. Human bodies are present inside where they are exposed to high-power electromagnetic radiation issued from the antenna. We applied the developed PLRC-FDTD method for the study of the electromagnetic waves' behavior in this case and to compute the human bodies' exposure to those radiations through the evaluation of the specific absorption rate (SAR).

\section{Formulations}

2.1. Four-Pole Debye Model. With the aim of modeling the response of the human body issues to the electromagnetic radiation at a broad band of microwave frequencies, the measured permittivity and conductivity data for each tissue [14] are approximated by a fourth-order Debye dispersive model, as expressed in the following equation:

$$
\varepsilon_{\mathrm{r}}(j \omega)=\varepsilon_{\mathrm{r}}^{\prime}(j \omega)+\varepsilon_{\mathrm{r}}^{\prime \prime}(j \omega)=\varepsilon_{\mathrm{\infty}}+\sum_{k=1}^{K} \frac{\Delta \varepsilon_{k}}{1+j \omega \tau_{k}}+\frac{\sigma_{\mathrm{s}}}{j \omega \varepsilon_{0}},
$$

where $\omega$ is the angular frequency, $\varepsilon_{0}$ is the free space permittivity, $\varepsilon_{\mathrm{r}}^{\prime}$ and $\varepsilon_{\mathrm{r}}^{\prime \prime}=\sigma /\left(\omega \varepsilon_{0}\right)$ are the real part and the imaginary part of the complex relative permittivity $\left(\varepsilon_{\mathrm{r}}\right)$ of the Debye dispersive media where $\sigma$ denotes the electric conductivity, $\varepsilon_{\infty}$ is the permittivity at the infinite frequency, $K$ is the number of Debye modes, $\Delta \varepsilon_{k}$ and $\tau_{k}$ are, respectively, the magnitude and the relaxation time of the $k$ th Debye dispersion mode, and $\sigma_{\mathrm{s}}$ is the static conductivity.

2.2. The Particle Swarm Optimization. In this paper, the Matlab toolbox based on the particle swarm optimization (PSO) algorithm is used to accurately fit the Debye parameterized model with a set of measured data [14], by minimizing the cost function $\zeta$ which is the mean of the relative errors between the data measurements and the Debye computed model at each measured frequency, as expressed in the following equation [10].

$$
\zeta=\frac{1}{2 N_{f}} \sum_{k=1}^{N_{f}}\left[\left|\frac{\varepsilon_{\mathrm{r}, \exp }\left(f_{k}\right)-\varepsilon_{\mathrm{r}, \text { Debye }}\left(f_{k}\right)}{\varepsilon_{\mathrm{r}, \exp }\left(f_{k}\right)}\right|+\left|\frac{\sigma_{\exp }\left(f_{k}\right)-\sigma_{\text {Debye }}\left(f_{k}\right)}{\sigma_{\exp }\left(f_{k}\right)}\right|\right] \text {, }
$$

where $\varepsilon_{\text {r,Debye }}$, and $\sigma_{\text {Debye }}$ are, respectively, the computed permittivity and conductivity using the Debye model. $\varepsilon_{\mathrm{r} \text { exp }}$ and $\sigma_{\exp }$ are the measured permittivity and conductivity, respectively [14].

The application of the particle swarm algorithm implies the minimization of the cost function $\zeta$. The experimental data [14] of 19 tissues are fitted over the frequency ranging from $100 \mathrm{MHz}$ to $3 \mathrm{GHz}$ with the fourthorder Debye model.

The PSO algorithm's flow involves first the creation of a random swarm of 2000 particles. Each particle has a position which is represented by a set of Debye parameters $\left[\varepsilon_{\infty}, \Delta \varepsilon_{1}\right.$, $\left.\tau_{1}, \ldots, \Delta \varepsilon_{4}, \tau_{4}, \sigma_{\mathrm{s}}\right]$ and a velocity which is the used to compute the next position. As a constraint, all the parameters are nonnegative numbers. At each iteration of the PSO algorithm, the cost function is evaluated at all particles to record the location of the best particle in the neighborhood of each particle and the best one over all particles. For each 
TABle 1: Parameters of the four-pole Debye model of human tissues.

\begin{tabular}{lccccccccccc}
\hline Tissues & Blood & Bone & Cerebellum & Fat & Heart & Kidney & Liver & Lung & Muscle & $\begin{array}{c}\text { White } \\
\text { matter }\end{array}$ \\
\hline$\varepsilon_{\infty}$ & $1.96 E+01$ & $7.11 E+00$ & $1.58 E+01$ & $2.07 E+00$ & $1.80 E+01$ & $1.80 E+01$ & $1.46 E+01$ & $6.68 E+00$ & $2.11 E+01$ & $1.81 E+01$ \\
$\Delta \varepsilon_{1}$ & $1.76 E+04$ & $5.38 E+04$ & $2.74 E+01$ & $1.74 E+04$ & $2.07 E+06$ & $1.03 E+05$ & $1.35 E+04$ & $9.21 E+06$ & $6.67 E+05$ & $1.03 E+04$ \\
$\Delta \varepsilon_{2}$ & $4.59 E+03$ & $2.52 E+02$ & $3.40 E+01$ & $1.09 E+01$ & $1.57 E+03$ & $1.32 E+03$ & $4.42 E+02$ & $4.62 E+02$ & $1.95 E+03$ & $1.93 E+02$ \\
$\Delta \varepsilon_{3}$ & $1.17 E+02$ & $2.99 E+01$ & $2.05 E+02$ & $2.56 E+00$ & $1.74 E+02$ & $3.72 E+01$ & $3.10 E+01$ & $6.85 E+01$ & $4.70 E+01$ & $1.96 E+01$ \\
$\Delta \varepsilon_{4}$ & $3.70 E+01$ & $1.16 E+01$ & $1.22 E+03$ & $1.51 E+00$ & $4.05 E+01$ & $2.55 E+02$ & $5.07 E+01$ & $1.42 E+01$ & $3.77 E+01$ & $4.11 E+01$ \\
$\tau_{1}[\mathrm{rad} / \mathrm{s}]$ & $8.65 E-04$ & $1.49 E-05$ & $9.98 E-04$ & $6.79 E-06$ & $5.48 E-05$ & $3.17 E-06$ & $5.22 E-07$ & $9.17 E-04$ & $1.13 E-05$ & $7.68 E-07$ \\
$\tau_{2}[\mathrm{rad} / \mathrm{s}]$ & $9.73 E-08$ & $4.97 E-08$ & $1.37 E-11$ & $9.92 E-09$ & $6.04 E-08$ & $4.81 E-08$ & $2.13 E-08$ & $4.37 E-08$ & $1.14 E-07$ & $1.35 E-08$ \\
$\tau_{3}[\mathrm{rad} / \mathrm{s}]$ & $5.86 E-09$ & $1.76 E-09$ & $3.50 E-09$ & $7.51 E-12$ & $4.45 E-09$ & $1.56 E-11$ & $1.64 E-11$ & $4.17 E-09$ & $1.37 E-09$ & $2.23 E-11$ \\
$\tau_{4}[\mathrm{rad} / \mathrm{s}]$ & $1.41 E-11$ & $1.68 E-11$ & $4.56 E-08$ & $8.82 E-10$ & $1.52 E-11$ & $4.20 E-09$ & $1.72 E-09$ & $1.41 E-11$ & $1.68 E-11$ & $1.27 E-09$ \\
$\sigma_{\mathrm{s}}[\mathrm{S} / \mathrm{m}]$ & 1.064 & 0 & $1.13 E-01$ & 0 & 0 & 0 & 0 & 0 & 0 \\
Errors & & & & & & & & & & & \\
Debye2 (PSO) & $4.91 E-03$ & $4.02 E-02$ & $1.27 E-02$ & $8.12 E-02$ & $1.17 E-02$ & $1.15 E-02$ & $1.96 E-02$ & $1.01 E-02$ & $2.78 E-02$ & $2.43 E-02$ \\
Debye4 (PSO) & $1.30 E-03$ & $6.05 E-03$ & $2.04 E-03$ & $4.10 E-03$ & $2.98 E-03$ & $2.57 E-03$ & $2.55 E-03$ & $2.01 E-03$ & $3.41 E-03$ & $4.56 E-03$ \\
Debye4 (GA) & $1.61 E-02$ & $8.58 E-03$ & $4.52 E-02$ & $2.52 E-02$ & $2.79 E-02$ & $2.66 E-01$ & $7.21 E-02$ & $4.01 E-03$ & $2.61 E-02$ & $3.54 E-02$ \\
Cole-Cole (PSO) & $1.80 E-04$ & $3.87 E-04$ & $4.98 E-04$ & $5.09 E-03$ & $1.23 E-04$ & $4.03 E-04$ & $4.16 E-04$ & $1.40 E-04$ & $2.13 E-04$ & $2.31 E-03$ \\
\hline
\end{tabular}



$$
\begin{array}{ll}
. \quad \text { Experimental } & - \text { Debye4 (GA) } \\
\triangle & \text { Debey2 (PSO) } \\
\rightarrow \text { Debye4 (PSO) } & -*-\text { Cole-Cole (PSO) }
\end{array}
$$

(a)

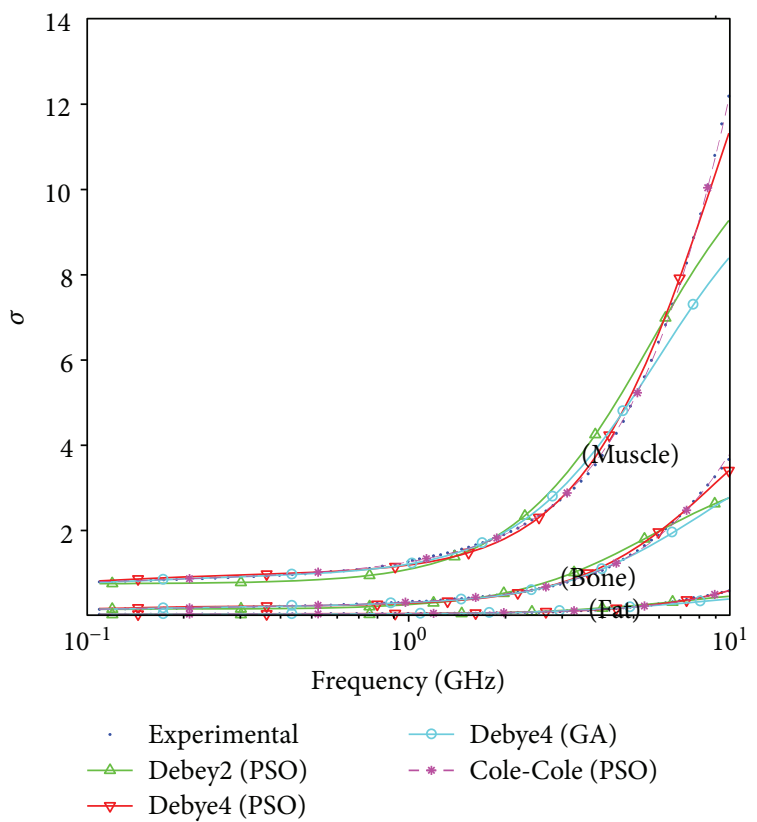

(b)

Figure 1: Properties of human body tissues using Debye model compared with the experimental data. (a) Relative permittivity. (b) Conductivity.

particle, the neighborhood is the first 500 nearest particles. The velocity of each particle is updated as a weighted sum of the previous velocity and the position of the neighborhood best particle and the global best particle. Then, the position of each particle is updated as a function of the previous position and the velocity. Over consecutive iterations, the swarm of particles converge toward the optimal solution with the lowest cost function. The maximum number of iterations is 10,000 .
For the aim of comparison, the genetic algorithm is performed in such a manner to have the same resource consumption as the PSO algorithm. The population size is 2000. The maximum number of generations is 10,000 .

Table 1 lists the four-pole Debye parameters of each tissue (due to the limited space, only a few of them are listed) and the evaluated errors of each model expressed by (2). The fitting results of some tissues are plotted in Figure 1. 
From Figure 1 and Table 1, it is shown that the Cole-Cole model offers the best agreement with the experimental data [7], then the four-pole Debye model computed by using the PSO algorithm offers almost the same performance as the Cole-Cole model, then the two-pole Debye offers lower fitting with the experimental data. In addition, if we consider the same model, the PSO algorithm offers more performance than the Genetic algorithm.

2.3. Formulation of the PLRC-FDTD. To investigate the electromagnetic wave behavior through a Debye dispersive media, the relationship relating the electric field intensity $\vec{E}$ to the electric induction $\vec{D}$ by the frequency-dependent permittivity $\varepsilon(\omega)$ is converted to the time domain by using the inverse Fourier transform.

$$
\begin{aligned}
D(t) & =\varepsilon(t) * E(t), \\
\varepsilon(t) & =\varepsilon_{0} \varepsilon_{\infty} \delta(t)+\left(\varepsilon_{0} \sum_{k=1}^{K} \frac{\Delta \varepsilon_{k}}{\tau_{k}} e^{-t / \tau_{k}}+\sigma_{\mathrm{s}}\right) U(t),
\end{aligned}
$$

where ${ }^{*}$ is the convolutional product, $\delta(t)$ is the Dirac delta function and $U$ is the unit step function. The substitution of $\varepsilon(t)$ in (3) yields the following:

$$
\begin{aligned}
D(t) & =\varepsilon_{0} \varepsilon_{\mathrm{\infty}} E(t)+\sum_{k=1}^{K} P_{k}(t)+P_{\text {loss }}(t), \\
P_{k}(t) & =\varepsilon_{0} \frac{\Delta \varepsilon_{k}}{\tau_{k}} \int_{0}^{t} e^{-(t-\tau) / \tau_{k}} E(\tau) d \tau, \\
P_{\text {loss }}(t) & =\sigma_{s} \int_{0}^{t} E(\tau) d \tau,
\end{aligned}
$$

where $P_{k}$ represents the polarization density of the $k$ th Debye mode. $P_{\text {loss }}$ is conductive loss term.

In the discretized mode, the polarization density and the conductive loss become

$$
\begin{aligned}
P_{k}^{n \Delta t}= & \varepsilon_{0} \frac{\Delta \varepsilon_{k}}{\tau_{k}} e^{-n \Delta t / \tau_{k}} \int_{0}^{(n-1) \Delta t} e^{\tau / \tau_{k}} E(\tau) d \tau \\
& +\varepsilon_{0} \frac{\Delta \varepsilon_{k}}{\tau_{k}} e^{-n \Delta t / \tau_{k}} \int_{(n-1) \Delta t}^{n \Delta t} e^{\tau / \tau_{k}} E(\tau) d \tau, \\
P_{\text {loss }}^{n \Delta t}= & \sigma_{\mathrm{s}} \int_{(n-1) \Delta t}^{n \Delta t} E(\tau) d \tau+\sigma_{\mathrm{s}} \int_{0}^{(n-1) \Delta t} E(\tau) d \tau .
\end{aligned}
$$

$P_{k}^{n \Delta t}$ and $P_{\text {loss }}^{n \Delta t}$ in (8) can be computed by a recursive formula:

$$
\begin{aligned}
& P_{k}^{n \Delta t}=e^{-\Delta t / \tau_{k}} P_{k}^{(n-1) \Delta t}+\varepsilon_{0} \frac{\Delta \varepsilon_{k}}{\tau_{k}} e^{-n \Delta t / \tau_{k}} \int_{(n-1) \Delta t}^{n \Delta t} e^{\tau / \tau_{k}} E(\tau) d \tau, \\
& P_{\text {loss }}^{n \Delta t}=\sigma_{\mathrm{s}} \int_{(n-1) \Delta t}^{n \Delta t} E(\tau) d \tau+P_{\text {loss }}^{(n-1) \Delta t} .
\end{aligned}
$$

The PLRC method is based on assuming that the electric field follows a piecewise linear behavior within each discretization interval, then it can be expressed as follows.

$$
E(t)=E^{(n-1) \Delta t}+\left(\frac{E^{n \Delta t}-E^{(n-1) \Delta t}}{\Delta t}\right)(t-(n-1) \Delta t) .
$$

By the substitution of (10) into the (9), the expression of the polarization density and the conductive loss become as follows.

$$
\begin{aligned}
& P_{k}^{n \Delta t}=e^{-\Delta t / \tau_{k}} P_{k}^{(n-1) \Delta t}+A_{k} E^{(n-1) \Delta t}+B_{k} E^{n \Delta t}, \\
& P_{\text {loss }}^{n \Delta t}=P_{\text {loss }}^{(n-1) \Delta t}+\frac{\sigma_{\mathrm{s}} \Delta t}{2}\left(E^{(n-1) \Delta t}+E^{n \Delta t}\right), \\
& P_{\text {loss }}^{n \Delta t}=P_{k}^{(n-1) \Delta t}+\frac{\sigma_{\mathrm{s}} \Delta t}{2}\left(E^{(n-1) \Delta t}+E^{n \Delta t}\right),
\end{aligned}
$$

where

$$
\begin{aligned}
& A_{k}=-\varepsilon_{0} \Delta \varepsilon_{k}\left(e^{-\Delta t / \tau_{k}}-\frac{\tau_{k}}{\Delta t}\left(1-e^{-\Delta t / \tau_{k}}\right)\right), \\
& B_{k}=\varepsilon_{0} \Delta \varepsilon_{k}\left(1-\frac{\tau_{k}}{\Delta t}\left(1-e^{-\Delta t / \tau_{k}}\right)\right) .
\end{aligned}
$$

By solving (5) after substitution of (11), the updated equation of the electric field intensity is resumed as follows:

$$
E^{n \Delta t}=\frac{1}{a}\left[D^{n \Delta t}+b E^{(n-1) \Delta t}-\sum_{k=1}^{K}\left(e^{-\Delta t / \tau_{k}} P_{k}^{(n-1) \Delta t}\right)-P_{\text {loss }}^{(n-1) \Delta t}\right],
$$

where

$$
\begin{aligned}
& a=\varepsilon_{0}\left(\varepsilon_{\infty}+\sum_{k=1}^{K} \Delta \varepsilon_{k}\left(1-\frac{\tau_{k}}{\Delta t}\left(1-e^{-\Delta t / \tau_{k}}\right)\right)+\frac{\sigma_{s} \Delta t}{2 \varepsilon_{0}}\right), \\
& b=\varepsilon_{0}\left(\sum_{k=1}^{K} \Delta \varepsilon_{k}\left(e^{-\Delta t / \tau_{k}}-\frac{\tau_{k}}{\Delta t}\left(1-e^{-\Delta t / \tau_{k}}\right)\right)-\frac{\sigma_{s} \Delta t}{2 \varepsilon_{0}}\right) .
\end{aligned}
$$

The advantage of using PLRC technique compared to the convolution-based FDTD method [10] resides in increasing the accuracy, by assuming that the electric field follows a piecewise linear function of the time, whereas the convolution-based method considers it as a constant in every discretization interval.

2.4. Validation. For the validation issues, a simple EM problem for which the analytical solution is well known is solved using the present method. Then, the results are compared with the analytical solution. This EM problem consists of a TEM Gaussian-pulse plane wave which is propagating along the $x$-direction and reflected by an air-dispersive media plane interface, where the chosen Debye parameters are those characterizing the fat in Table 1. The computational environment is surrounded by a PEC boundary condition from the upside and the downside, and a PMC boundary from the left and right sides, and ten cells of perfectly matched layer (PML) from the front and back sides, as shown in Figure 2. The 


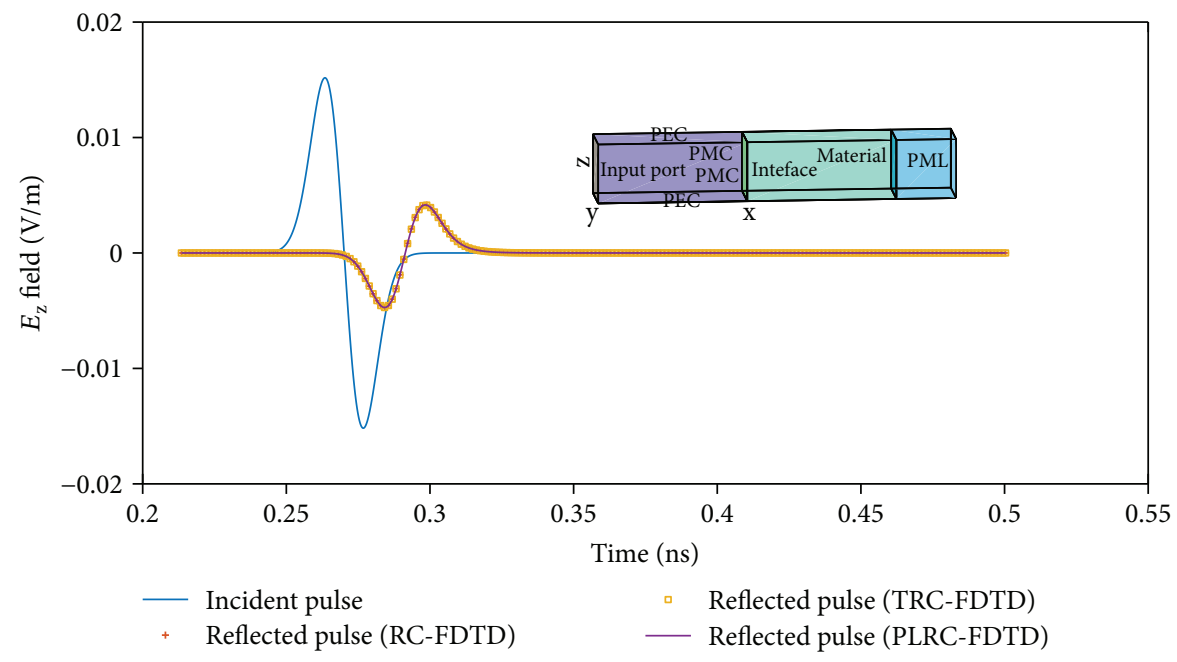

Figure 2: Plane wave normal incident on a vacuum/Debye model interface.

Gaussian pulse is parameterized in such a way to cover the frequency band of interest $(100 \mathrm{MHz}-3 \mathrm{GHz})$. A uniform Yee grid is used with a space increment of $4 \mathrm{~mm}$, which provides a minimum resolution of 25 cells per wavelength (at $3 \mathrm{GHz}$ ). Figure 2 shows the incident and the reflected wave from the interface in the time domain. The reflection coefficient computed by the PLRC, TRC, and RC methods is compared with the analytical solution in the frequency domain as shown in Figure 3(a). The relative error calculated using (15), which evaluates the accuracy of the used numerical methods, is shown in Figure 3(b).

$$
\operatorname{Err}=20 \log \left(\left|\frac{\Gamma_{\text {num }}-\Gamma_{\text {ana }}}{\Gamma_{\text {ana }}}\right|\right),
$$

where $\Gamma_{\text {ana }}$ is the analytically computed reflection coefficient and $\Gamma_{\text {num }}$ is that computed numerically by using one of the techniques: PRLC, TRC, or RC.

Figure 2 shows that the three methods present an excellent agreement with the analytical solution. However, both PLRC and TRC methods demonstrate a lower error compared to the RC method along the frequency band of interest as shown in Figure 3. This is explained by the accuracy order of each method, where PLRC and TRC methods are of second-order accuracy, and the RC is of first order.

\section{Simulation Results}

A military vehicle (Humvee) with a mounted antenna on its rear part is modeled by using a reduced complexity model. The complexity is reduced by eliminating the geometrical details which are far smaller than the smallest studied wavelength. Then, a crew composed of one, two, or four people is added. The geometrical characteristics of the human bodies are acquired from a large dataset of human tissue model [15] which is evaluated and accepted by the IEEE International Committee on Electromagnetic Safety for the calculation of the specific absorption rates. All the geometric entities are converted from vertex data to voxel model which is appropriate to the FDTD grid as shown in Figure 4. Each crew member is represented by a heterogeneous phantom which is formed by taking into consideration the geometrical characteristics of all the organs in [15]. Then, the phantoms are postured in a seating position by using geometrical transformation for the limbs.

The radiating antenna is fed with a wideband Gaussian pulse covering a broad frequency band from $0.1 \mathrm{GHz}$ to $3 \mathrm{GHz}$. The computational domain is $x=5.4400 \mathrm{~m}$ in length, $y=2.86 \mathrm{~m}$ in width, and $z=3.5 \mathrm{~m}$ in height. In order to allow the simulation for an open space, a perfectly matched layer (PML) of 20 cells surrounds the computational space. The simulation is carried out for the sufficient time allowing the total attenuation of the electromagnetic waves. The space discretization increment is $\Delta x=\Delta y=\Delta z=4 \mathrm{~mm}$, which provides a minimum resolution of 25 points per wavelength, which is sufficient for obtaining accurate results as proven in Section 2.4.

The simulation is performed by using the Matlab parallel computing toolbox. The electric and magnetic flux densities are represented by $1360 \times 715 \times 875$ sized threedimensional variables covering all the computational space, whereas the electric and the magnetic field intensity are computed just in the nonvacuum space. The simulation requires 41 Go of memory with an average of 55 octets by cell. The FDTD algorithm is run for 10,000 iterations, the equivalent time of $50 \mathrm{~ns}$ which is the necessary time for the electromagnetic waves to vanish throughout the PML absorbing boundary. In order to showcase the electric field distribution at various frequencies, we applied the discrete Fourier transformation for each cell in the computational environment.

First, we consider the case of a crew composed of four people. The human body tissues exposure to electromagnetic radiation is evaluated by the within-tissue electric field strength as reported in Figure 5 which shows the computed distribution of the electric field intensity in an arbitrary chosen vertical slice passing through two passengers at the frequency of $200 \mathrm{MHz}$. It is clear that the 


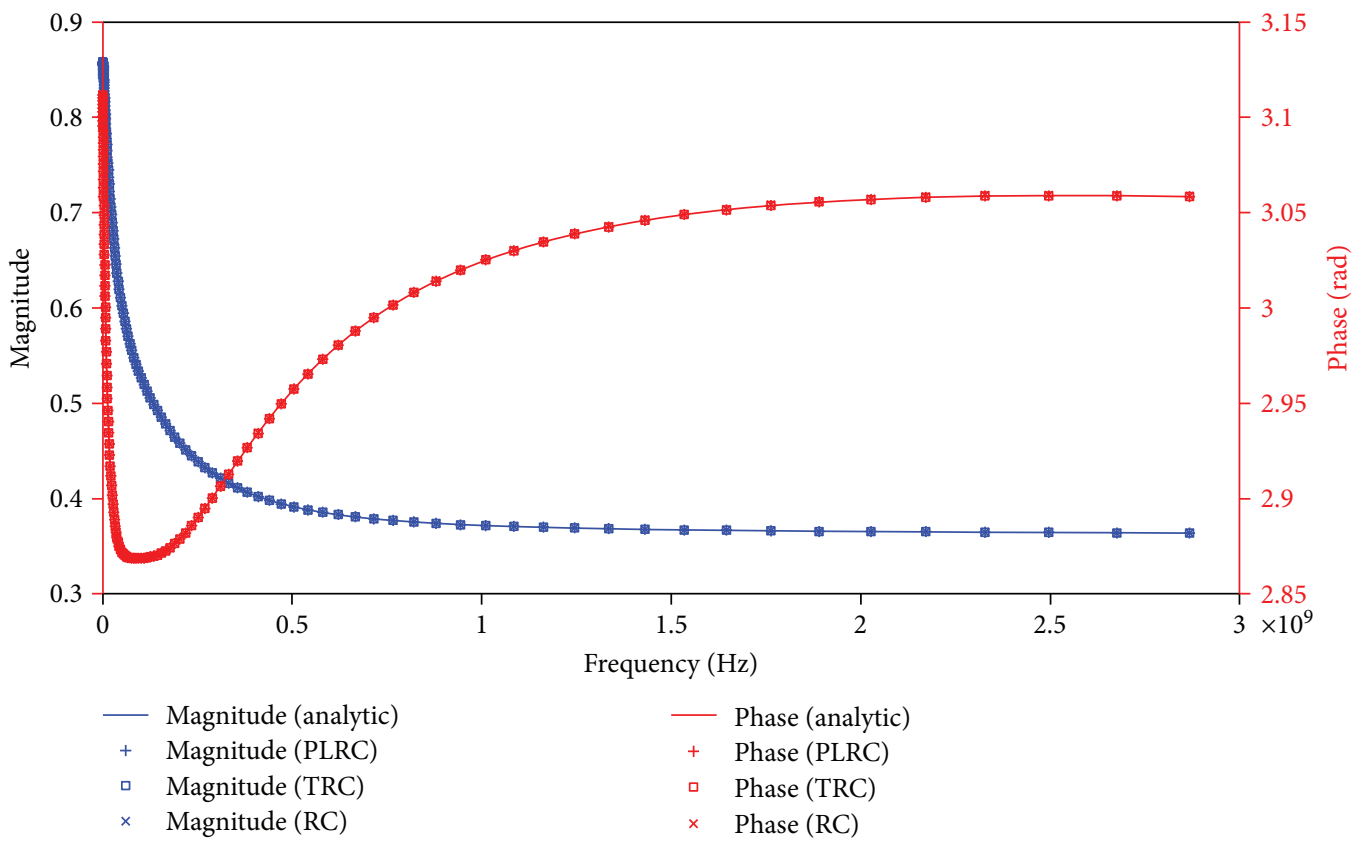

(a)

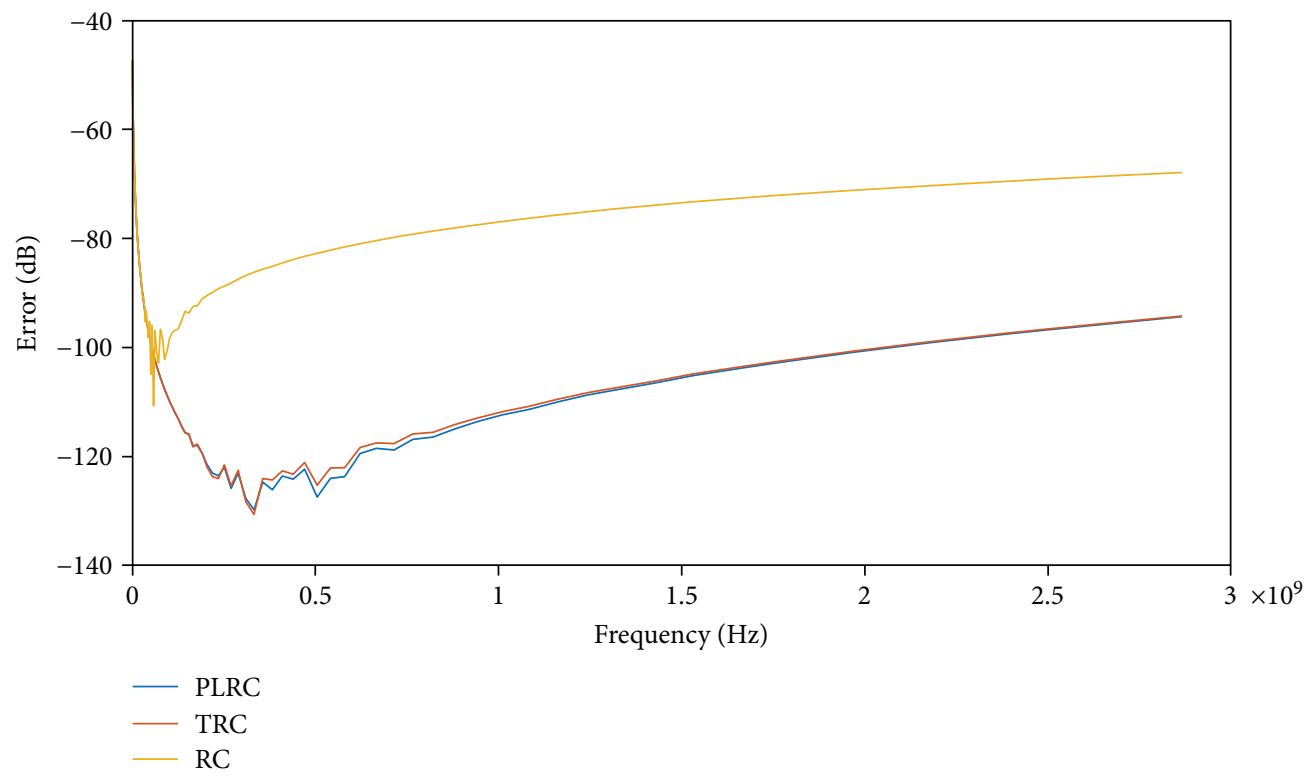

(b)

FIgURE 3: Comparison of the computed reflection coefficient by RC, TRC, and PLRC techniques with the theoretical values. (a) Magnitude and phase. (b) Relative errors.

electric field intensity in the vacuum is higher than that inside the human body. This fact is due to the conductor aspect of the human tissues.

Then, to perform an electromagnetic resonance analysis of the human body, particular locations in the human body have been chosen as detection points (head, heart, and ankle) as marked in Figure 5 by white marks. Figure 6 shows the spectrum of the electric field strength at the marked points. We notice the existence of resonance modes at the interval $100 \mathrm{MHz}-1 \mathrm{GHz}$, depending on the geometry of each part of the body and the dielectric characteristics of its tissue.
For the brain, the main resonance frequency is observed at $0.25 \mathrm{GHz}$; for the heart and the ankle, the main resonant frequency is $0.6 \mathrm{GHz}$.

Second, the simulation is repeated for the cases of a crew composed of one and two occupants. The computed electromagnetic dosimetry is compared with the recommended dosimetry reference limits (DRLs) established by the IEEE standard for the military workspaces [2], where different local SAR limits are identified for some regions of the body. In [2], the environments are classified into two classes: unrestricted zones, where the access is allowed for all personnel 




FIgURE 4: 3D model of the Humvee with four human bodies inside.

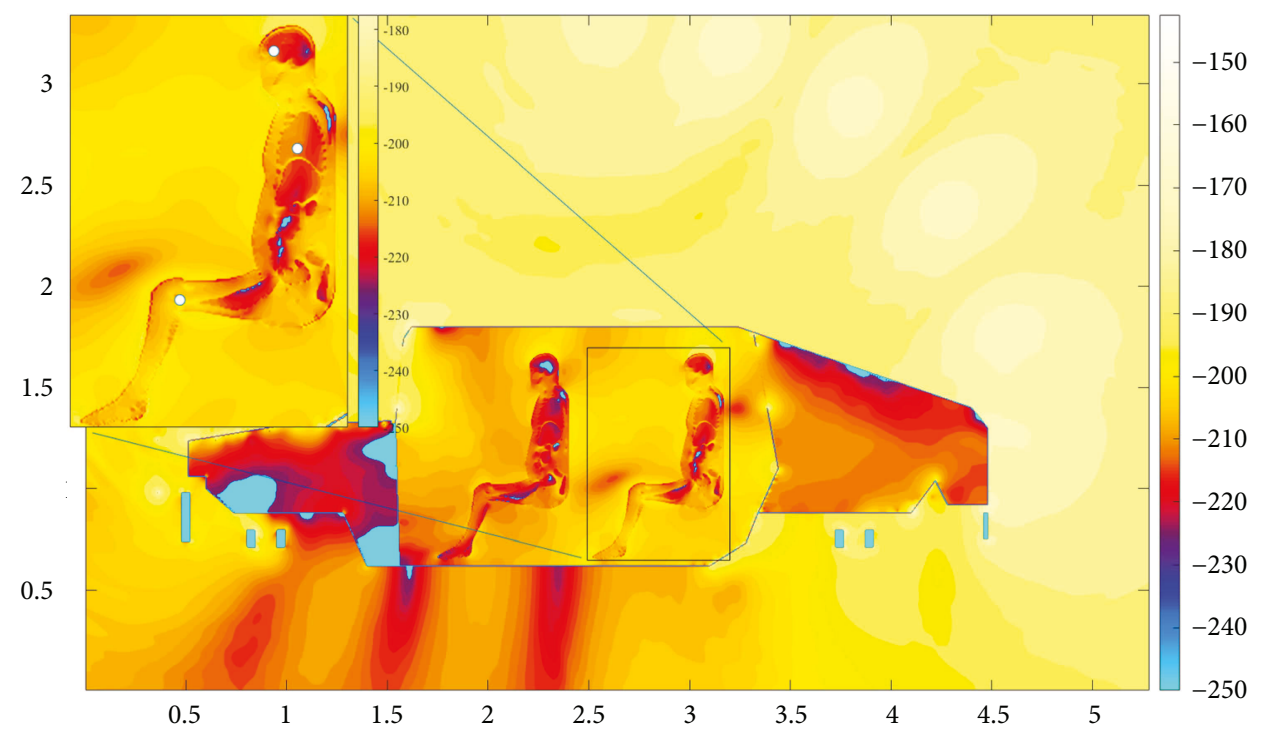

FIGURE 5: Electric field distribution at the frequency $200 \mathrm{MHz}$.

and the DRLs are strict; and restricted zones, where only the informed personnel are authorized to access and the DRLs are more relaxed than the unrestricted areas. For unrestricted environments, the standard [2] defines the limits for the whole-body average SAR as $0.08 \mathrm{~W} / \mathrm{kg}$, the $10 \mathrm{~g}$ local SAR in the head and the trunk as $2 \mathrm{~W} / \mathrm{kg}$, and the local SAR for the arms and legs as $4 \mathrm{~W} / \mathrm{kg}$.

Table 2 summarizes the maximum values of the computed SAR for a normalized radiated power of $1 \mathrm{~W}$ $\mathrm{CW}$, and the frequencies for which those maximums are detected, for one, two, and four members of the crew and for each occupant in the crew $(1,2,3$, and 4 denote the driver, front passenger, left rear passenger, and rear right passenger, resp.).

The obtained whole-body average and local SAR values for a radiation power of $1 \mathrm{~W}$ are significantly lower than the recommended limits. Since the computed SAR values are proportional to the radiated power, those values can be scaled to a higher level without exceeding the recommended limits.

From the obtained results, it is clear that the SAR level depends on the total number of occupants. For example, if 


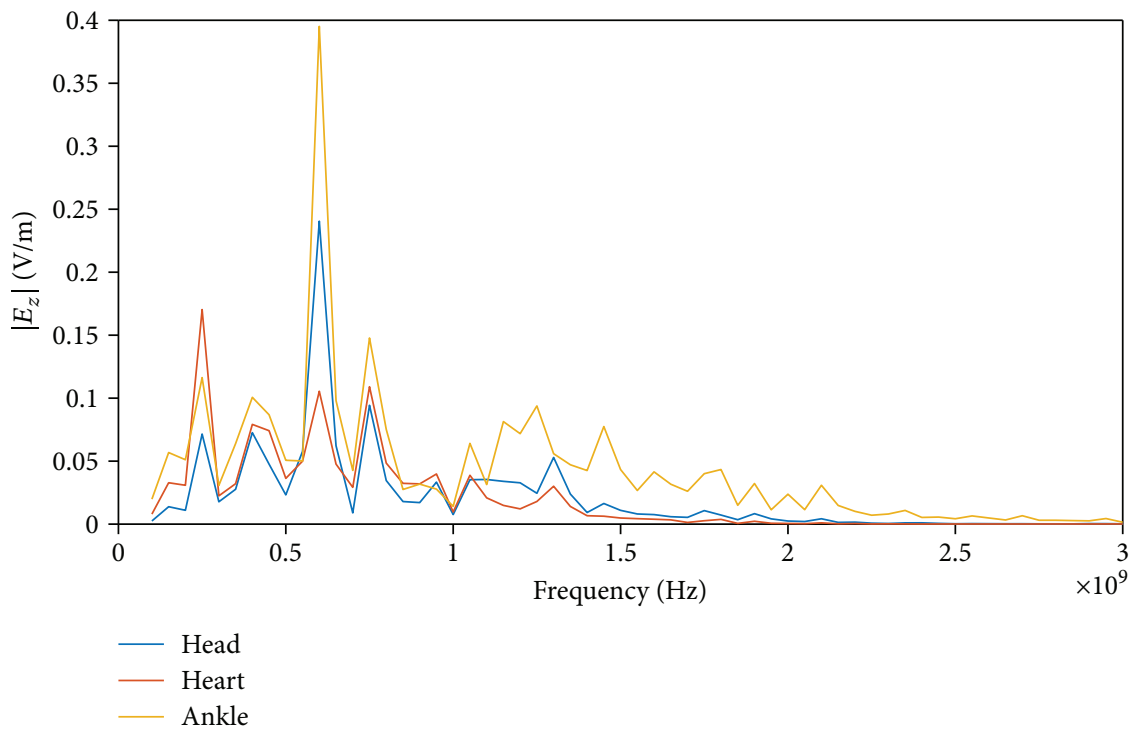

Figure 6: Electric field strength at the head, the heart, and the ankle of the 3rd occupant.

TABLE 2: Computed SAR For AT $1 \mathrm{~W}$ CW.

\begin{tabular}{|c|c|c|c|c|c|}
\hline SAR measure & & Occupant 1 & Occupant 2 & Occupant 3 & Occupant 4 \\
\hline \multicolumn{6}{|l|}{ One occupant } \\
\hline \multirow{2}{*}{ Whole-body average } & Maximum $(\mathrm{W} / \mathrm{kg})$ & $4.174 E-05$ & & & \\
\hline & Frequency $(\mathrm{MHz})$ & 600 & & & \\
\hline \multirow{2}{*}{ Local SAR for head and trunk } & Maximum $(\mathrm{W} / \mathrm{kg})$ & $1.236 E-03$ & & & \\
\hline & Frequency $(\mathrm{MHz})$ & 600 & & & \\
\hline \multirow{2}{*}{ Local SAR for limbs } & Maximum (W/kg) & $1.362 E-03$ & & & \\
\hline & Frequency $(\mathrm{MHz})$ & 600 & & & \\
\hline \multicolumn{6}{|l|}{ Two occupants } \\
\hline \multirow{2}{*}{ Whole-body average } & Maximum $(\mathrm{W} / \mathrm{kg})$ & $2.904 E-05$ & $2.062 E-05$ & & \\
\hline & Frequency $(\mathrm{MHz})$ & 600 & 600 & & \\
\hline \multirow{2}{*}{ Local SAR for head and trunk } & Maximum $(\mathrm{W} / \mathrm{kg})$ & $1.015 E-03$ & $8.671 E-04$ & & \\
\hline & Frequency $(\mathrm{MHz})$ & 600 & 600 & & \\
\hline \multirow{2}{*}{ Local SAR for limbs } & Maximum $(\mathrm{W} / \mathrm{kg})$ & $1.260 E-03$ & $5.757 E-04$ & & \\
\hline & Frequency $(\mathrm{MHz})$ & 600 & 600 & & \\
\hline \multicolumn{6}{|l|}{ Four occupants } \\
\hline \multirow{2}{*}{ Whole-body average } & Maximum (W/kg) & $1.59 E-05$ & $8.82 E-06$ & $2.92 E-05$ & $8.30 E-06$ \\
\hline & Frequency $(\mathrm{MHz})$ & 600 & 600 & 600 & 600 \\
\hline \multirow{2}{*}{ Local SAR for head and trunk } & Maximum (W/kg) & $4.674 E-4$ & $7.417 E-4$ & $8.606 E-4$ & $3.277 E-4$ \\
\hline & Frequency $(\mathrm{MHz})$ & 650 & 600 & 600 & 675 \\
\hline \multirow{2}{*}{ Local SAR for limbs } & Maximum (W/kg) & $7.433 E-4$ & $5.775 E-4$ & $1.207 E-4$ & $4.388 E-4$ \\
\hline & Frequency $(\mathrm{MHz})$ & 675 & 600 & 600 & 600 \\
\hline
\end{tabular}

we consider the first occupant, the SAR level is most important in the case of a single occupant. Also, we notice that in the case of four occupant crew, the left rear occupant is more affected by the antenna radiation than the other occupants. This can be explained by the fact that this occupant is nearest to the antenna.

According to those results, the maximum radiation power for which the SAR limits might not be attained is
$1.62 \mathrm{KW}$, which is commonly greater than the usually used power for military operations on this type of vehicles.

\section{Conclusion}

In this paper, an efficient 3D PLRC-FDTD method is developed to provide an accurate simulation of the electromagnetic wave propagation in the human body to better 
assess the specific absorption rates in environments characterized by high levels of radiation.

The PLRC-FDTD was used for the simulation of a $3 D$ voxel model of the military vehicle (Humvee) with a mounted transmission antenna in the presence of human bodies. The particle swarm optimization was used to derive four-pole Debye models for the whole-body tissues in the frequency band from $0.1 \mathrm{GHz}$ to $3 \mathrm{G} \mathrm{Hz}$. In addition to its accurate matching with the experimental electric characteristics of the human tissues, the Debye model also offers an ease of implementation.

This allows the computation of the in-tissue electromagnetic field's intensity and consequently the calculation of the specific absorbing rate than the localization of regions with a high exposure to electromagnetic radiation in a broad band of frequency. The PLRC method, which is second-order accuracy, fulfills considerably improved precision over the RC method.

The computed SAR levels for $1 \mathrm{~W}$ CW radiated power are significantly lower than the recommended limits. These results also demonstrate that those limits cannot be attended unless the radiated power exceeds $1.62 \mathrm{~kW}$, which is uncommon regarding the typically used equipment in the military vehicle.

\section{Conflicts of Interest}

The authors declare that there is no conflict of interest regarding the publication of this paper.

\section{Acknowledgments}

This project is partly supported by the National Natural Science Foundation of China (Grant no. 61371044).

\section{References}

[1] D. Garrido, M. Ignatenko, and D. S. Filipovic, "Computational study of electromagnetic exposure of military personnel in a Humvee," in 2014 United States National Committee of URSI National Radio Science Meeting (USNC-URSI NRSM), p. 1, Boulder, CO, USA, 2014.

[2] "IEEE standard for military workplaces-force health protection regarding personnel exposure to electric, magnetic, and electromagnetic fields, $0 \mathrm{~Hz}$ to $300 \mathrm{GHz}$," in IEEE Std C95.12345-2014, pp. 1-69, New York, NY, USA, 2014, IEEE.

[3] A. R. Ruddle, "Computed SAR levels in vehicle occupants due to on-board transmissions at $900 \mathrm{MHz}$," in 2009 Loughborough Antennas \& Propagation Conference, pp. 137-140, Loughborough, UK, 2009, IEEE.

[4] G. Anzaldi, F. Silva, M. Fernandez, M. Quilez, and P. J. Riu, "Initial analysis of SAR from a cell phone inside a vehicle by numerical computation," IEEE Transactions on Biomedical Engineering, vol. 54, no. 5, pp. 921-930, 2007.

[5] Y. Diao, "SAR evaluation for multiple wireless communication devices inside a vehicle," in 2013 International Symposium on Electromagnetic Theory, pp. 626-629, Hiroshima, Japan, 2013.

[6] A. R. Ruddle, L. Low, J. M. Rigelsford, and R. J. Langley, "Variation of computed in-vehicle SAR with number and location of occupants at commonly used communications frequencies," in 10th International Symposium on Electromagnetic Compatibility, pp. 756-761, York, UK, 2011.

[7] S. Gabriel, R. W. Lau, and C. Gabriel, “The dielectric properties of biological tissues: III. Parametric models for the dielectric spectrum of tissues," Physics in Medicine \& Biology, vol. 41, no. 11, pp. 2271-2293, 1996.

[8] T. Wuren, T. Takai, M. Fujii, and I. Sakagami, "Effective 2Debye-pole FDTD model of electromagnetic interaction between whole human body and UWB radiation," IEEE Microwave and Wireless Components Letters, vol. 17, no. 7, pp. 483-485, 2007.

[9] M. Fujii, R. Fujii, R. Yotsuki, T. Wuren, T. Takai, and I. Sakagami, "Exploration of whole human body and UWB radiation interaction by efficient and accurate two-Debyepole tissue models," IEEE Transactions on Antennas and Propagation, vol. 58, no. 2, pp. 515-524, 2010.

[10] S. Mustafa, A. M. Abbosh, and P. T. Nguyen, "Modeling human head tissues using fourth-order Debye model in convolution-based three-dimensional finite-difference timedomain," IEEE Transactions on Antennas and Propagation, vol. 62, no. 3, pp. 1354-1361, 2014.

[11] S. G. Garcia, R. G. Rubio, A. R. Bretones, and R. G. Martin, "Extension of the ADI-FDTD method to debye media," IEEE Transactions on Antennas and Propagation, vol. 51, no. 11, pp. 3183-3186, 2003.

[12] J. Shibayama, R. Ando, A. Nomura, J. Yamauchi, and H. Nakano, "Simple trapezoidal recursive convolution technique for the frequency-dependent FDTD analysis of a Drude-Lorentz model," IEEE Photonics Technology Letters, vol. 21, no. 2, pp. 100-102, 2009.

[13] I. Giannakis and A. Giannopoulos, "A novel piecewise linear recursive convolution approach for dispersive media using the finite-difference time-domain method," IEEE Transactions on Antennas and Propagation, vol. 62, no. 5, pp. 2669-2678, 2014.

[14] C. Gabriel, Compilation of the Dielectric Properties of Body Tissues at RF and Microwave Frequencies, Radiofrequency Radiation Division, Brooks Air Force Base, San Antonio, TX, USA, 1996, http://www.dtic.mil/cgi-bin/GetTRDoc? $\mathrm{AD}=\mathrm{ADA} 309764$.

[15] S. Makarov, G. Noetscher, and A. Nazarian, Low-frequency electromagnetic modeling for electrical and biological systems using MATLAB, Wiley, Hoboken, NJ, USA, 2016. 


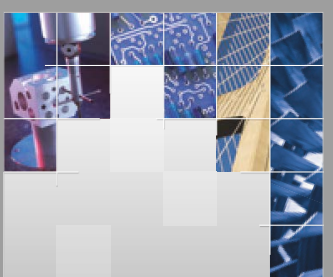

\section{Enfincering}


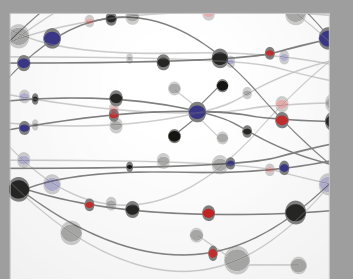

\section{Rotating \\ Machinery}

The Scientific World Journal

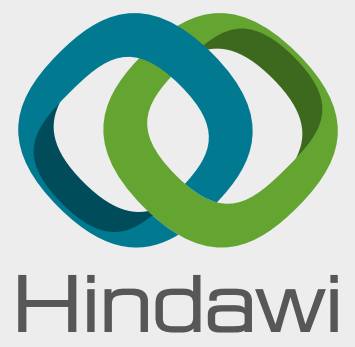

Submit your manuscripts at

www.hindawi.com
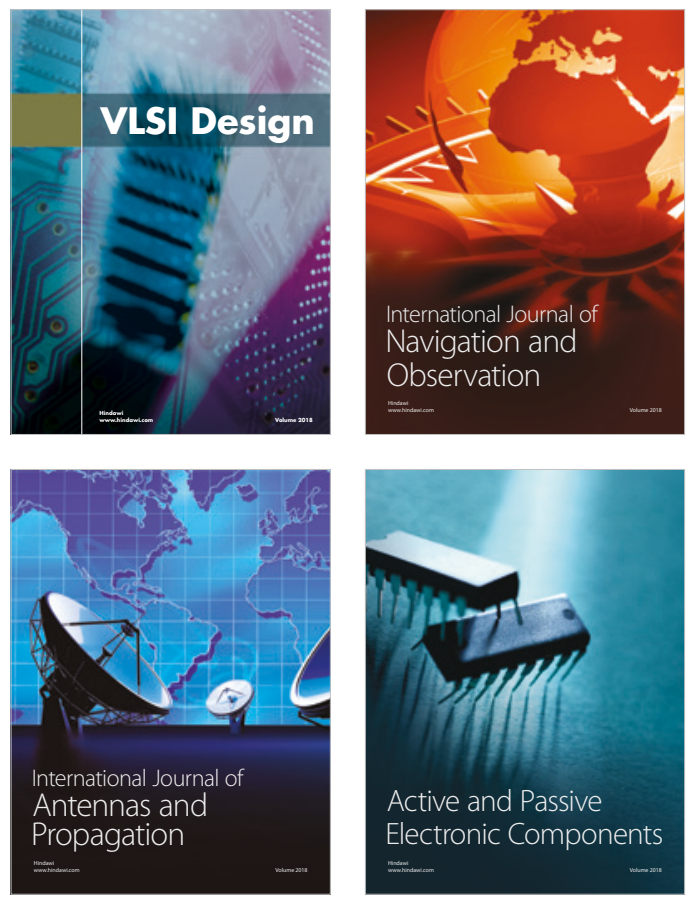
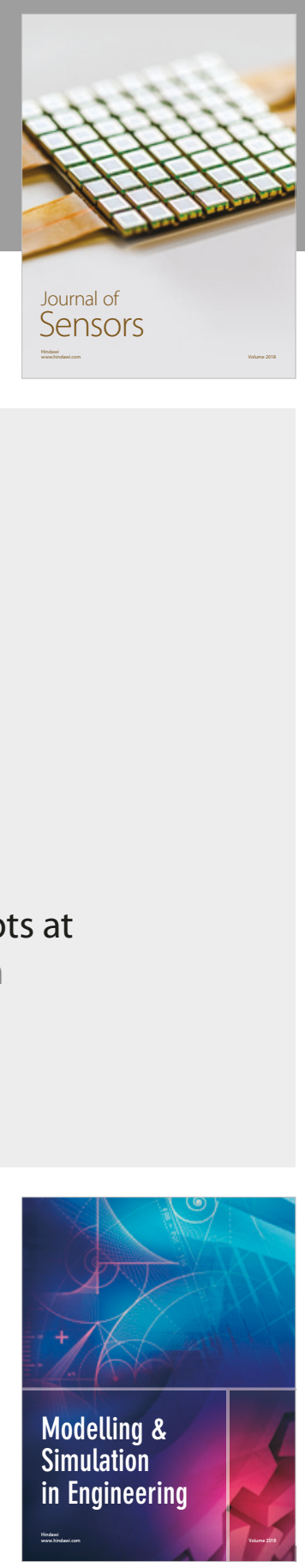

\section{Advances \\ Multimedia}
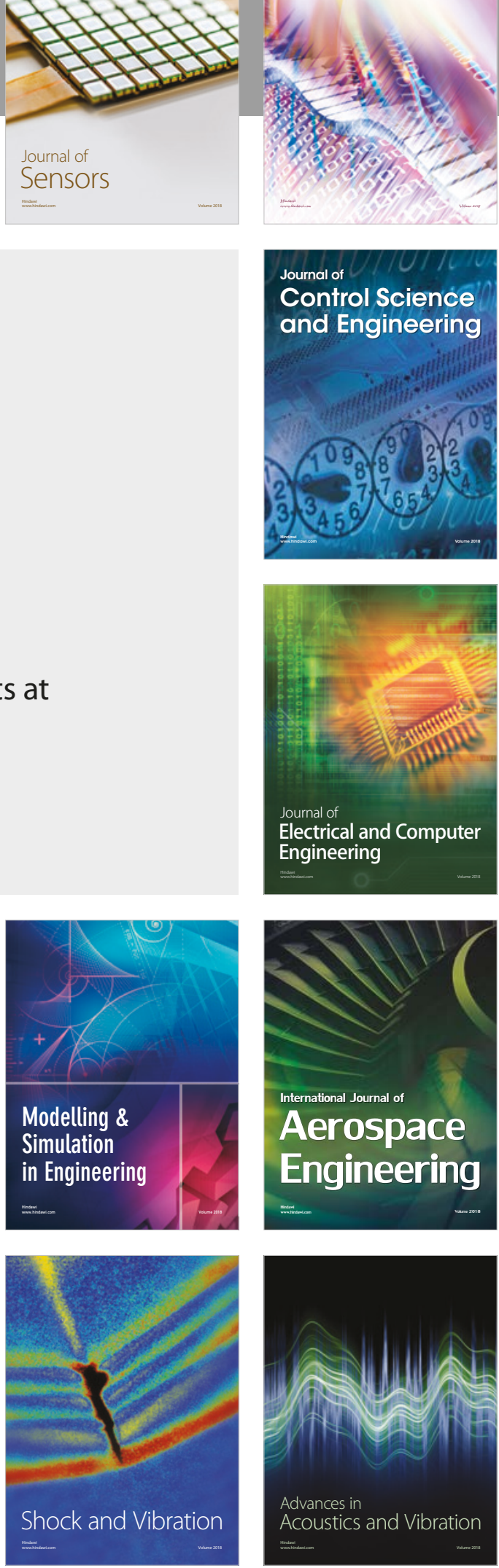